\title{
Electronic Structure and Magnetovolume Instabilities of the Hexagonal Laves Phase Compound $\mathrm{Fe}_{2} \mathrm{Ti}$
}

\author{
E. Hoffmann, P. Entel, E. Wassermann*, K. Schwarz** and P. Mohn** \\ Theoretische Tieftemperaturphysik, Gerhard-Mercator-Universität, Gesamthochschule Duisburg, \\ 47048 Duisburg, Germany \\ * Laboratorium für Tieftemperaturphysik, Gerhard-Mercator-Universität, Gesamthochschule Duisburg, \\ 47048 Duisburg, Germany \\ ** Institut für Elektrochemie, Technische Universität Wien, 1060 Wien, Austria
}

\begin{abstract}
We investigate the electronic and cohesive properties of the hexagonal and cubic Laves phases of $\mathrm{Fe}_{2} \mathrm{Ti}$ by ab initio band structure calculations. In particular the stability range of these compounds is studied. In the volume range considered, the state of lowest energy is hexagonal (C14) and exhibits antiferromagnetic order. However, we find a slightly higher in energy lying ferromagnetic state with low magnetic moment (smaller than $1 \mu_{B}$ ). Interestingly enough, this moment can considerably be enhanced (larger than $1.5 \mu_{B}$ ) for larger lattice spacings. The characteristic moment-volume dependence of the Fe atom can also account for the experimental observation that $\mathrm{Fe}_{2+x} \mathrm{Ti}_{1-x}$ is ferromagnetic for $\mathrm{x}>0$ since the smaller Fe atom leads to a contraction of the lattice which stabilizes large ferromagnetic iron clusters.
\end{abstract}

\section{Introduction}

We have shown in Ref. $[1,2]$ that in iron-rich FeNi compounds the close relationship between magnetovolume instabilities (Invar) and martensitic behaviour is responsible for all physical properties of this material. Here we study magnetovolume instabilities in the $\mathrm{Fe}_{2} \mathrm{Ti}$ system hoping to find similar trends as in FeNi. Experimental investigations of $\mathrm{Fe}_{2+x} \mathrm{Ti}_{1-x}$ have shown that there is a rich spectrum of magnetic anomalies which are caused by mixing different ferromagnetic and antiferromagnetic states [3-5]. It has also been observed that the stoichiometric hexagonal Laves phase $\mathrm{Fe}_{2+x} \mathrm{Ti}_{1-x}$ for $\mathrm{x}=0$ separates regions $\mathrm{x}<0$ and $\mathrm{x}>0$ which are of appreciably different magnetic order. Increasing the iron concentration leads to mainly ferromagnetic order while increasing the titanium concentration leads to antiferromagnetic order. The ground state of $\mathrm{Fe}_{2} \mathrm{Ti}$ (C14) as deduced from experiment seems to be very close to a situation which corresponds to coexisting ferromagnetic and antiferromagnetic states. This is confirmed by our calculations. The $\mathrm{Fe}_{2} \mathrm{Ti}$ system has previously been investigated by
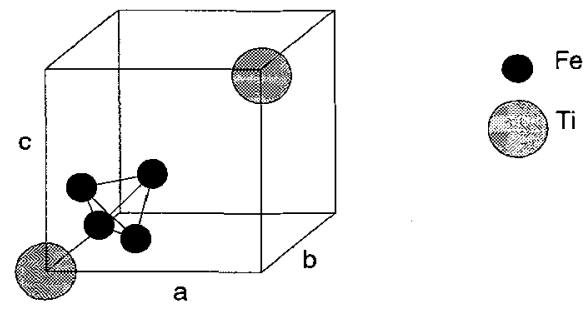

Figure 1: Unit cell of the cubic $\mathrm{Fe}_{2} \mathrm{Ti}$ Laves phase with $\mathrm{C} 15$ $\mathrm{MgCu}_{2}$ structure. The Bravais lattice of the system is fcc with $3 \mathrm{M}$ symmetry in (111) direction. 
Asano and Ishida $[6,7]$ with the LMTO method. However, they only investigated the ferromagnetic and antiferromagnetic equilibrium states and estimated their volume and magnetization dependence in a very crude way. We have studied the system again by performing first-principles Augmented Spherical Wave calculations [8] by using the nonrelativistic spin-polarized LDA approach of Janak [9]. This allows us to describe the ground state of the iron atom with sufficient accuracy [10]. In order to get a first impression of the importance of spin and volume (charge) fluctuations in the $\mathrm{Fe}_{2+x} \mathrm{Ti}_{1-x}$ system, we have also calculated the ferro- and antiferromagnetic binding surfaces of ordered $\mathrm{Fe}_{2} \mathrm{Ti}$. The C15 structure corresponds to an fcc-lattice with basis and $3 \mathrm{M}$ symmetry; the unit cell contains 2 formula units of $\mathrm{Fe}_{2} \mathrm{Ti}$. The cartesian coordinates of the basis atoms of the $\mathrm{C} 15$ structure (see Fig.1) are: Ti: 1) $(0,0,0), 2)\left(\frac{3}{4}, \frac{3}{4}, \frac{3}{4}\right)$, Fe: 1) $\left.\left.\left.\left(\frac{3}{8}, \frac{3}{8}, \frac{3}{8}\right), 2\right)\left(\frac{3}{8}, \frac{1}{8}, \frac{1}{8}\right), 3\right)\left(\frac{1}{8}, \frac{3}{8}, \frac{1}{8}\right), 4\right)\left(\frac{1}{8}, \frac{1}{8}, \frac{3}{8}\right)$.

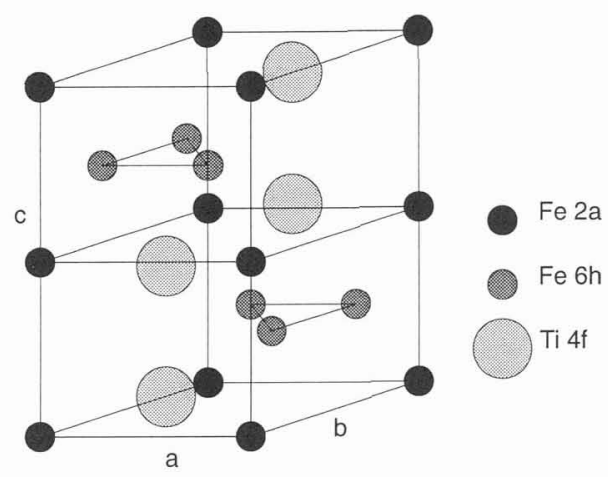

Figure 2: Unit cell of $\mathrm{Fe}_{2} \mathrm{Ti}$ with C14 $\mathrm{MgZn}_{2}$ structure. The intraplane [001] coupling of the $6 \mathrm{~h}$ iron moments is always ferromagnetic. The coupling between nearest $\mathrm{Fe}(6 \mathrm{~h})$ planes can be ferromagnetic or antiferromagnetic.

The hexagonal Laves phase contains 4 formula units in the unit cell. If we neglect magnetic ordering then there are three different types of sites in the lattice. One type of Ti site (4f) and two types of Fe sites $(2 a, 6 h)$. With respect to the Bravais vectors of the system, the coordinates of the Fe(2a) sites are: $\mathrm{Fe}(2 \mathrm{a}): 1)(0,0,0), 2)\left(0,0, \frac{1}{2}\right)$; the $\mathrm{Ti}(4 \mathrm{f})$ atoms are located at: 3$\left.)\left(\frac{1}{3}, \frac{2}{3}, z\right), 4\right)\left(\frac{1}{3}, \frac{2}{3}, \frac{1}{2}-z\right)$, 5) $\left.\left(\frac{2}{3}, \frac{1}{3}, \frac{1}{2}+z\right), 6\right)\left(\frac{2}{3}, \frac{1}{3}, c-z\right)$; the $\mathrm{Fe}(6 \mathrm{~h})$ sites build two planes in the lattice which are seperated by titanium and iron (2a) planes. The first plane is built by: 7) $\left.\left.\left(-x,-2 x, \frac{1}{4}\right), 8\right)\left(-x, x, \frac{1}{4}\right), 9\right)\left(2 x, x, \frac{1}{4}\right)$ and the second one by: 10) $\left.\left.\left(-2 x,-x, \frac{3}{4}\right), 11\right)\left(x, 2 x, \frac{3}{4}\right), 12\right)\left(x,-x, \frac{3}{4}\right)$ with $\mathrm{z}=\frac{1}{16}$ and $\mathrm{x}=-\frac{1}{6}$ for the ideal C14 structure. Both planes always couple ferromagnetically in plane, the interplane coupling can be both antiferromagnetic or ferromagnetic. Recent neutron scattering results obtained by Brown et al. [11] have shown that the $\mathrm{Ti}$ and $\mathrm{Fe}(6 \mathrm{~h})$ positions deviate slightly form their ideal positions. The measured values are $\mathrm{x}=-0.17052$ and $\mathrm{z}=0.0647$. We compared both, ideal and reconstructed C14 structures and found no significant differences. The calculations presented here have been done for the reconstructed structure with an ideal $c / a$ ratio for the hexagonal closed packed structures with $c / a=2 \sqrt{\frac{2}{3}}$.

The ratio of the iron and titanium Wigner-Seitz spheres has been optimized at the experimental lattice constant by searching for the lowest energy state. This yields $\mathrm{r}_{T i} / \mathrm{r}_{F e}=1.237$. In order to minimize the computational time for these calculations we have investigated systematically the error in $\mathrm{k}$-space integration when using different sets of $\mathrm{k}$ points. We found that we have to use at least $80 \mathrm{k}$ points for the $\mathrm{C} 14$ structure in order to be sure that the integration error is neglegible with respect to relevant energy differences between the different magnetic states. So we calculated the C14 structure with $105 \mathrm{k}$ points in the irreducible wedge of the hexagonal Brillouin zone and the C15 structure with $120 \mathrm{k}$ points in the irreducible wedge of the fcc Brillouin zone with $3 \mathrm{M}$ symmetry in (111) direction.

In order to study the magnetovolume coupling we performed fixed-spin-moment (FSM) calculations for the C15 structure by fixing the magnetic moment of the whole cell. For the C14 structure we have regarded two types of constraints: first, ferromagnetic order as for the C15 structure and second, antiferromagnetic order of the $\mathrm{Fe}(6 \mathrm{~h})$ plains in (001) direction. 


\section{Results}

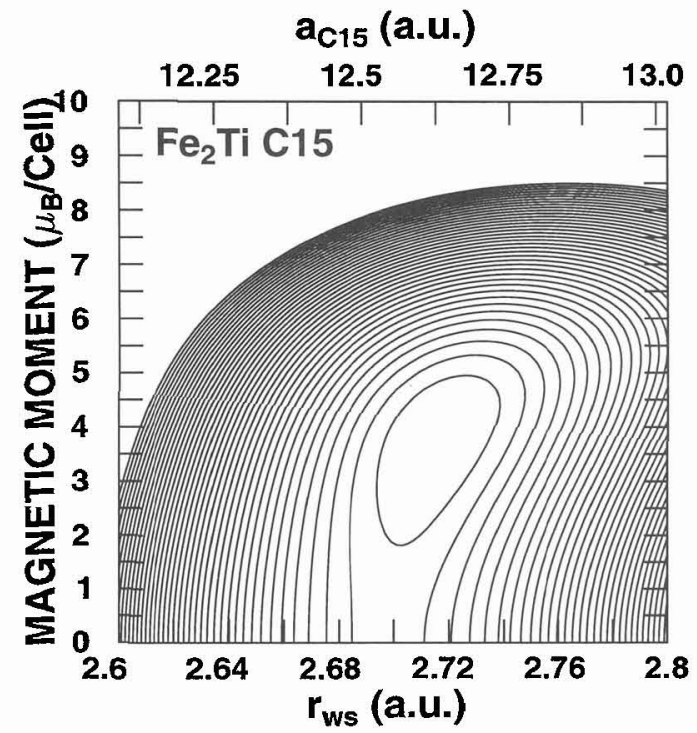

Figure 3: Binding surface of $\mathrm{Fe}_{2} \mathrm{Ti}$ in the C15 structure. Contour lines are at $0.25 \mathrm{mRy} /$ Atom intervals relative to the equilibrium energy. The equilibrium point at $\mathrm{M}=3.5 \mu_{B}$ and $\mathrm{r}_{W S}=2.713$ a.u. corresponds to the ferromagnetic ground state.

Figure 3 shows the binding surface of $\mathrm{Fe}_{2} \mathrm{Ti}$ with $\mathrm{C} 15$ structure. The effective Wigner-Seitz radius has been calculated by implying the condition that the volume of all spheres should be equal to the volume of the unit cell. Characteristics of this binding surface are common to many metallic ferromagnets. For example the great magnetic anharmonicity of the surface is an indication of magnetovolume instability and of Invar behaviour.

With respect to the symmetry there are 4 nonequivalent sites in the crystal. However, atomic properties of the different iron (titanium) sites are not distinguishable from each other as shown in

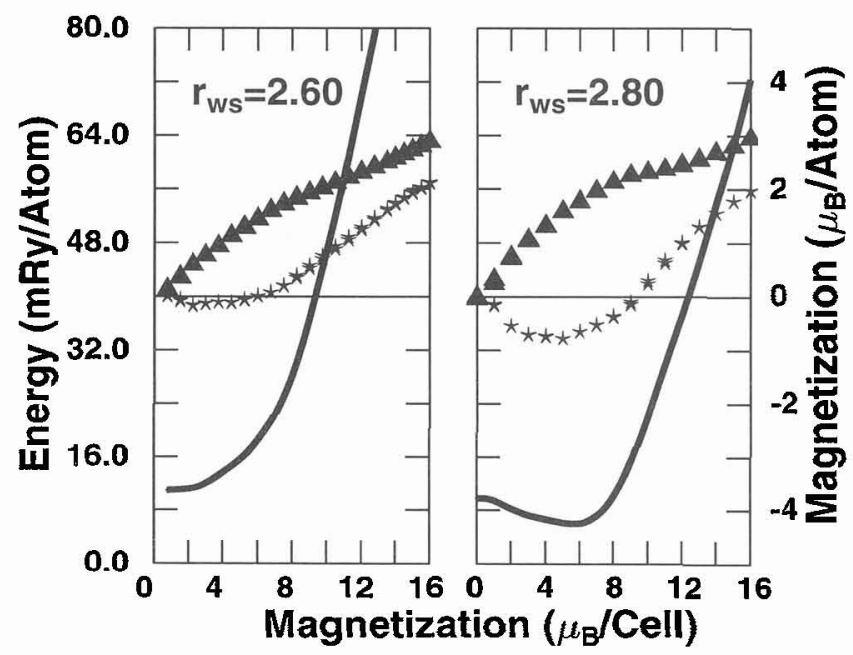

Figure 4: Binding energy and local magnetization of $\mathrm{Fe}_{2} \mathrm{Ti}$ in the $\mathrm{C} 15$ structure. The solid line shows the energy with respect to the ground state energy of the C14 structure. Triangles indicate the magnetic moment of the Fe atoms, stars the moment of the $\mathrm{Ti}$ atoms. 


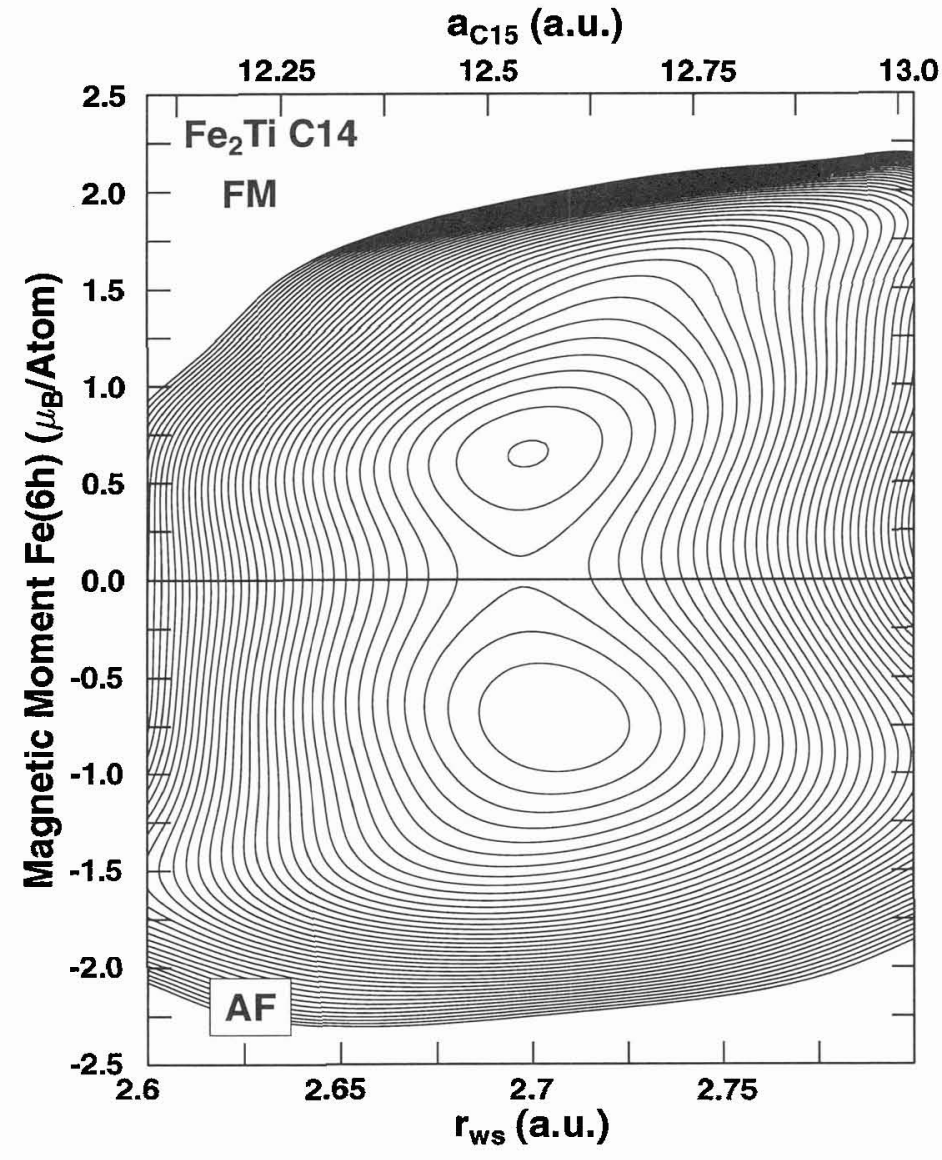

Figure 5: Binding surface of $\mathrm{Fe}_{2} \mathrm{Ti}$ in the C14 structure. Contour lines are at $0.25 \mathrm{mRy} /$ Atom intervals relative to the equilibrium energy. Positive magnetization of the $\mathrm{Fe}(6 \mathrm{~h})$ atoms means ferromagnetic ordering; negative magnetization denotes antiferromagnetic ordering of the $\mathrm{Fe}(6 \mathrm{~h})$ planes. The ground state is antiferromagnetically ordered with $\mathrm{M}=0.75 \quad \mu_{B}$ and $\mathbf{r}_{W S}=2.718$ a.u. The ferromagnetic state refers to $\mathrm{M}=0.59 \mu_{B}$ and $\mathrm{r}_{W S}=2.698$ a.u.

Figure 4. This Figure also shows that for large volumes and low magnetic moments the Ti atoms order antiparallel to the Fe atoms. This dominates the overall magnetic moment of the unit cell. The situation in the hexagonal Laves phase is somewhat more complicated because there are more possibilities of different kinds of magnetic ordering. Figure 5 shows the energy surface as function of the Wigner-Seitz radius and the magnetization of the $\mathrm{Fe}(6 \mathrm{~h})$ atoms. The real ground state is antiferromagnetically ordered but the ferromagnetic state lies only $0.1 \mathrm{mRy} /$ Atom higher in energy. This can be seen better in Figure 7. The softening of the AF state at high volume is also seen in the FM phase and is an indication for the crossover to local magnetic moment behaviour. Unusual is the softening at low volumes and magnetization of the $\mathrm{Fe}(6 \mathrm{~h})$ atoms around $1.5 \mu_{B}$. In Figure $6 \mathrm{a}$ we have plotted the magnetization of the $\mathrm{Fe}(2 \mathrm{a})$ and $\mathrm{Ti}(4 \mathrm{f})$ atoms. We show only the results for low volume, but the behaviour is quite the same in the whole volume range considered. It is obvious that the softening is related to the occurrence of a local magnetic moment of the Fe(2a) atoms. This indicates that we have not reached the limit of covalent paramagnetism in the considered volume range. The ferromagnetic state shows a typical low-spin to high-spin transition [12, 13]. At low volumes the equilibrium magnetization of the $\mathrm{Fe}(6 \mathrm{~h})$ atoms is about $0.75 \mu_{B}$. This state disappears at $r_{W S}=2.774$ a.u.; at $r_{W S}=2.764$ a.u. a high-spin state is stabilized with a magnetization of 1.6 $\mu_{B}$. The ferromagnetic phase shows a remarkable distribution of different local magnetic moments. The magnetization of the $\mathrm{Fe}(6 \mathrm{~h})$ atoms is always collinear with the average magnetization, but the $\mathrm{Fe}(2 \mathrm{a})$ and $\mathrm{Ti}(4 \mathrm{f})$ atoms have the tendency to order antiparallel to the Fe(6h) atoms (Figure $6 \mathrm{~b}$ ). This tendency increases with increasing volume. In Figure 7 we compare the different magnetic and structural states of $\mathrm{Fe}_{2} \mathrm{Ti}$. The most stable state is always the antiferromagnetic $\mathrm{C} 14$ phase, but the 


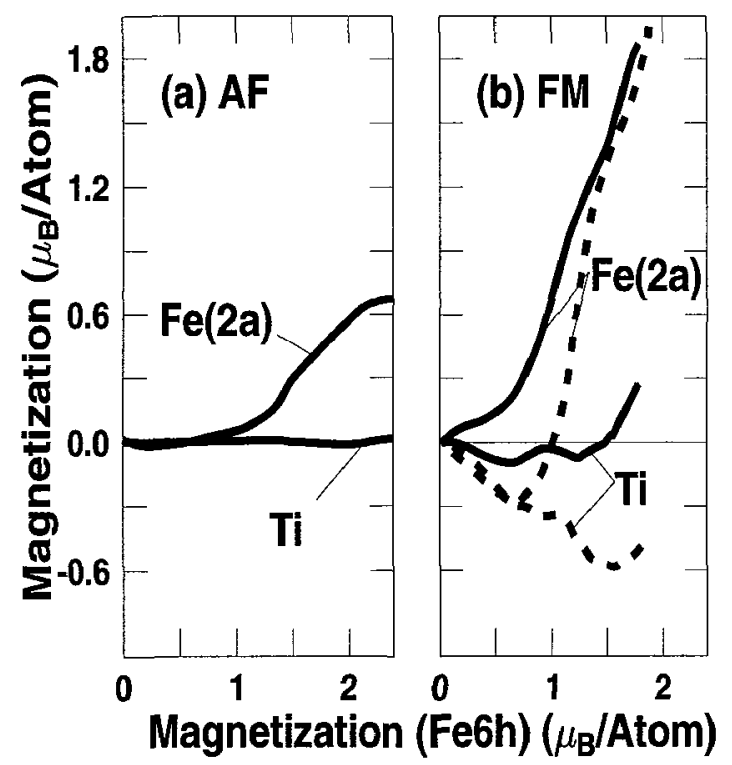

Figure 6: Local magnetization of the $\mathrm{Fe}(2 \mathrm{a})$ and $\mathrm{Ti}(4 \mathrm{f})$ atoms of $\mathrm{Fe}_{2} \mathrm{Ti}$ in the C14 structure as function of the $\mathrm{Fe}(6 \mathrm{~h})$ magnetization. Fig. $6 \mathrm{a}$ shows the magnetization for the antiferromagnetic case with $\mathrm{r}_{W S}=2.60$. Fig. $6 \mathrm{~b}$ shows the local magnetic moment for the ferromagnetic case for low volume (solid line) and high volume (dashed line). The Wigner Seitz radii are: 2.60 and 2.76 a.u., respectively.

exitation energies to the nonmagnetic and ferromagnetic states as well as the excitation energies from the $\mathrm{C} 14$ to the C15 structure are very small and comparable to the energy associated with thermal fluctuations of the order of $\mathrm{k}_{B} \mathrm{~T}_{N}$ with $\mathrm{T}_{N}=275 \mathrm{~K}$ for $\mathrm{Fe}_{2} \mathrm{Ti}$.

\section{Discussion}

The behaviour of the ferromagnetic high-spin state in the C14 structure is in contradiction to the results of Asano ond Ishida [7]. They found that the high-spin state is stable in the whole volume range and that it is energetically prefered at high volume. In their calculations floating magnetization

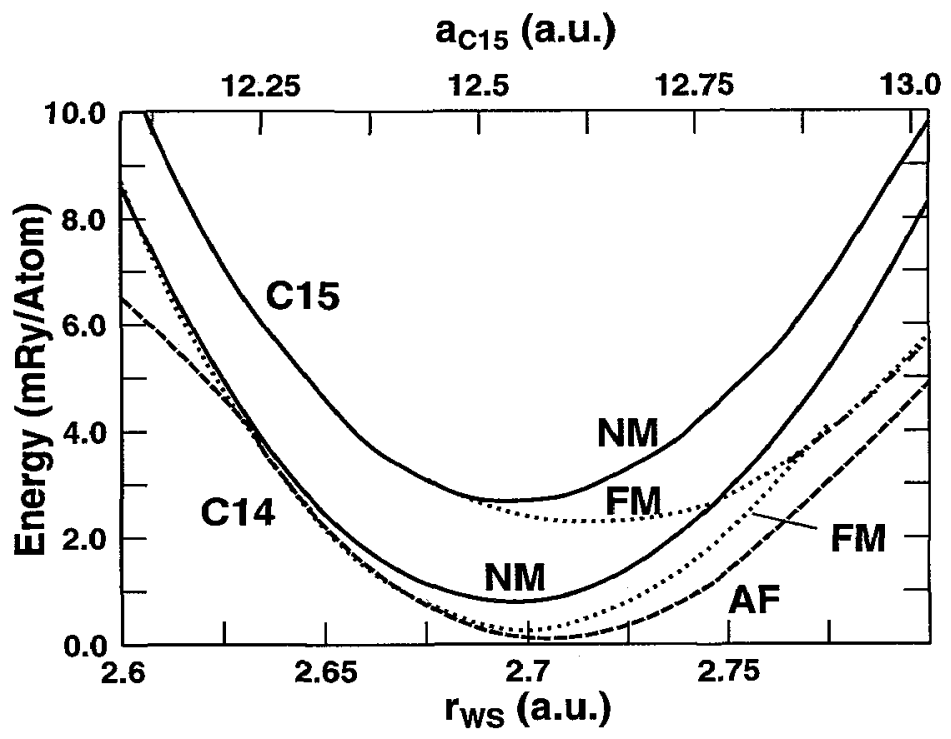

Figure 7: Binding energy of $\mathrm{Fe}_{2} \mathrm{Ti}$ in the $\mathrm{C} 14$ and C15 structure. 
was taken into account only in a Stoner-like way by introducing a variable potential difference $V_{\sigma}=V_{0}+\alpha \Delta V$ for the spin-polarized band structure. They also neglected the antiferromagnetic behaviour of the $\mathrm{Fe}(2 \mathrm{a})$ and $\mathrm{Ti}(4 \mathrm{f})$ atoms. In our calculation we used the FSM method [14] which simulates an internal field by introducing different Fermi-levels for the spin-up and down electronic states. With this method it is unlikely to generate nonphysical equilibrium states. Because of the energy differences between different magnetic and structural phases of $\mathrm{Fe}_{2} \mathrm{Ti}$ being very small and because of the tendency of antiferromagnetic ordering it would be very promising to perform noncollinear spin-structure calculations. This is left for the future.

\section{References}

[1] Entel P., Hoffmann E., Mohn P., Schwarz K. and Moruzzi V.L., Phys. Rev. B 47 (1993) 87068720.

[2] Hoffmann E., Herper H., Mishra S.G., Mohn P. and Schwarz K., Phys. Rev. B 47 (1993) 55895596.

[3] Muraoka Y., Shiga M. and Nakamura Y., J. Phys. Soc. Japan Letters 40 (1976) 905.

[4] Nishihara Y., J. Mag. Magn. Mat. 70 (1987) 75-80.

[5] Wassermann E.F., Rellinghaus B., Pasek M., Kriegel J., and Pepperhoff W., submitted to Phys. Rev. B.

[6] Asano S. and Ishida S., J. Magn. Magn. Mat. 70 (1987) 39-43.

[7] Asano S. and Ishida S., J. Phys.: Condens. Matter 1 (1989) 8501-8508.

[8] Williams A.R., Kübler J., and Gellat C.D., Phys. Rev. B 19 (1979) 6094-6118.

[9] Janak F., Phys. Rev. B 9 (1974) 3985.

[10] Moruzzi V.L., Marcus P.M., Schwarz K. and Mohn P., Phys. Rev. B 34 (1986) 1784-1791.

[11] Brown P.J., Deportes J. and Ouladdiaf B., J. Phys: Condens. Matter 4 (1992) 10015.

[12] Wassermann E.F., J. Magn. Magn. Mat. 100 (1991) 346-362.

[13] Acet M., Zähres H., Wassermann E.F. and Pepperhoff W., Phys. Rev. B 49 (1994) 6012-6017.

[14] Schwarz K. and Mohn P., J. Phys. F: Met. Phys. 14 (1984) L129-L134. 